Ann. Biol. anim. Bioch. Biophys., 1977, 17 (1), 33-41.

\title{
Durée du renouvellement de l'épithélium intestinal chez le porc adulte selon deux modes d'administration de la thymidine tritiée
}

\author{
par J. C. MESLIN, F. DABURON* \\ avec la collaboration technique de Y. TRICAUD* et de Nelly KAUSHIK** \\ Station de Recherches de Nutrition, I. N. R. A. \\ * Laboratoire de Radiobiologie Appliquée, C. E. A.-I. N. R. A. \\ ** Laboratoire de Nutrition des Poissons, I. N. R. A. \\ 78350 jouy-en-josas, France
}

Summary. Use of tritiated thymidine to study the renewal time of epithelial cells of adulf pig small intestine.

Four adult Pitman Moore pigs fitted with a permanent fistula of the proximal jejunum are used in this experiment. Tritiated thymidine is administered in two ways, by perfusion in the jugular vein and by injection into the intestinal lumen. The kinetics of the two animals are studied using a biopsy probe. Renewal time of small bowel epithelium is 4.5 in one pig and 5.5 days in the other. These two animals are used in further experiments to determine renewal time and the level of tritiated thymidine to be employed. A dose of $0.1 \mu \mathrm{Ci} / \mathrm{g}$ by perfusion is sufficient for good labelling of intestinal epithelium. Injecting very small quantitaties of radionuclide $(300 \mu \mathrm{Ci})$ intraluminally gives satisfactory autoradiographs after the slides are exposed 4.5 months.

\section{Introduction.}

L'étude du renouvellement de l'épithélium intestinal chez les gros animaux pose des problèmes pour l'expérimentation comparativement à celles effectuées classiquement sur les petits animaux de laboratoire. Le prix de revient de l'expérience, la quantiłé de radio-élément à manipuler en toute sécurité, la dose minimale compatible avec un bon marquage et une durée d'exposition des préparations non démesurément allongée sont autant de critères à faire intervenir.

Les seules données concernant le porc sont celles de Moon (1971) sur porcelets âgés respectivement de 1 et 21 jours et, à notre connaissance, aucune étude n'a été réalisée chez le porc adulte.

Moon et Joel (1975) ont étudié la migration des cellules épithéliales de l'intestin grêle chez le mouton à 1 jour, 3 semaines et 3 mois et chez le veau à 1 jour et 3 mois, 
par injection intraveineuse de 0,3 ou $0,7 \mu \mathrm{Ci}$ de thymidine tritiée par gramme de poids vif. Ceci conduit à utiliser des quantités très importantes de radioélément. Wachholz et Hopper (1971) ont démontré la possibilité de marquage des cellules de la muqueuse intestinale par administration intraluminale de thymidine tritiée. Chez l'embryon de poulet, la quantité de thymidine tritiée absorbée, après application locale, est fonction du temps de contact (Mawhinney, Austin et Riley, 1972).

Le but du présent travail est de déterminer la durée de renouvellement de l'épithélium intestinal chez le porc adulte, soit après perfusion dans la jugulaire, soit après injection dans la lumière intestinale de la thymidine tritiée. Dans ce dernier cas, on a faif varier la quantié de radio-élément administré ainsi que le temps de contact avec la muqueuse intestinale. Les différents animaux étudiés sont porteurs d'une fistule permanente de l'intestin. Pour chacune des conditions précitées, des prélèvements ont été effectués sur le même animal à différents intervalles de temps à l'aide d'une sonde à biopsie introduite par la fistule. Les techniques d'histo-autoradiographie ont permis de déterminer la position du front des cellules marquées en fonction du temps et d'en déduire la durée de renouvellement de l'épithélium intestinal dans les conditions de celte étude.

\section{Matériel et méthodes.}

Les animaux en expérience étaient porteurs d'une fistule intestinale permanente réalisée au moyen d'une canule rigide en acier inoxydable de $110 \mathrm{~mm}$ de long (fig. 1). Cette canule débouchait d'un côté dans la lumière du jéjunum proximal à $1 \mathrm{~m}$ environ de l'anse sigmoïde duodénale et de l'autre à travers la peau de la paroi abdominale, du côté droit, environ $10 \mathrm{~cm}$ en arrière de l'angle constitué par l'appendice xyphoïde et l'insertion sternale des dernières côtes.

Les biopsies, d'un poids de 5 à $10 \mathrm{mg}$, étaient prélevées chez les sujets légèrement anesthésiés à l'halothane, à l'aide d'une sonde gastro-intestinale à prélèvements de Quinton Instrument (sonde à succion dont l'extrémité est équipée d'un système de

PLANCHE I

FIG. 1. - Canule intestinale d̀ demeure permettant le passage des sondes à biopsie de muqueuse

FIG. 2. - Aspect d'une biopsie du jéjunum proximal chez le porc $\times 220$.

Coloration en masse par le carmin boracique 
PI. I
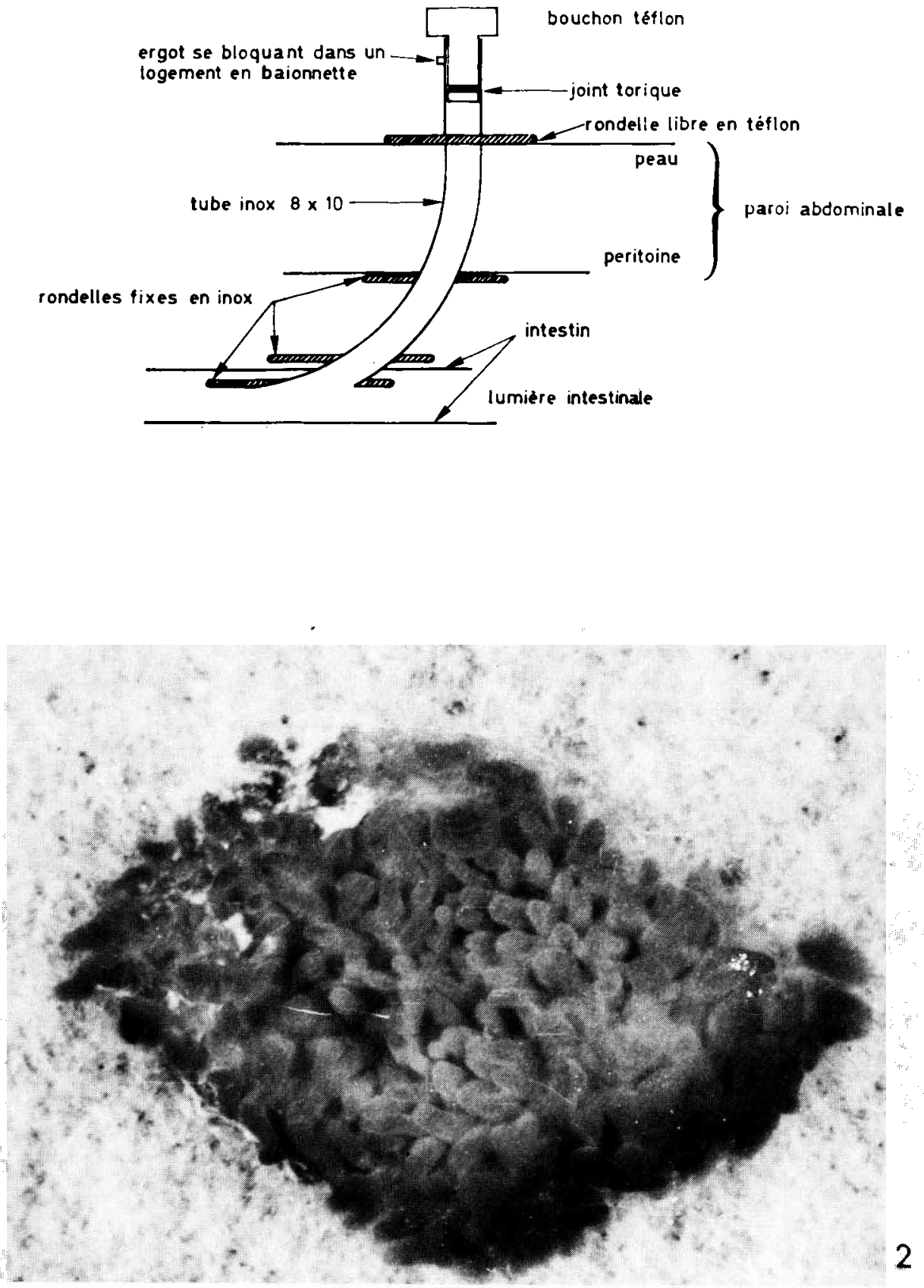
guillotine) : celle-ci était poussée à travers la canule, dont la courbure facilitait le cheminement, de 40 à $60 \mathrm{~cm}$ dans la lumière intestinale.

La sonde à deux ballonnets, utilisés pour le marquage intraluminal avait les caractéristiques suivantes: Sonde en caoutchouc du type «Sonde pour cavographie sélective du Dr Gillot» - Fabricant : J. Eynard et Cie, Paris-6e. Longueur, $90 \mathrm{~cm}$; Diamètre des ballonnets gonflés, $2,5 \mathrm{~cm}$; Distance entre les ballonnets, $30 \mathrm{~cm}$; Nombre de trous entre les ballonnets, 7 ; Calibre 15 Charrière $(5 \mathrm{~mm})$.

Les 4 animaux utilisés sont des porcs de race Pitman Moore adultes, âgés d'un an ef de poids moyen de $55 \mathrm{~kg}$. Ils sont soumis à un régime normal, sous forme de granulés, dont la composition est la suivante : cellulose, 5,7 p. 100 ; lipides, 3,2 p. 100 ; matières azotées, 16,7 p. 100 ; matières minérales, 6,2 p. 100. Cet aliment est distribué à raison d'un $\mathrm{kg}$ à chaque repas. Les prélèvements qui seront effectués le matin correspondent à des animaux à jeun depuis $18 \mathrm{~h}$ et ceux effectués le soir, à $7 \mathrm{~h}$ après le repas du matin.

- Le porc 1 a reçu $5 \mathrm{mCi}$ (environ $0,1 \mu \mathrm{Ci} / \mathrm{g}$, soit une dose dix fois moindre que celle utilisée chez le rat) de thymidine méthyle ${ }^{3} \mathrm{H}$ (A.S. $44 \mathrm{Ci} / \mathrm{mM}$ ) en solution dans $250 \mathrm{ml}$ de sérum physiologique glucosé iso-osmotique par perfusion dans la jugulaire. La perfusion a duré $2 \mathrm{~h}$. Des biopsies ont été prélevées dans le premier quart du jéjunum $24,31,48,55$ et $72 \mathrm{~h}$ après la fin de la perfusion.

- Le porc 2 a reçu, au niveau du premier quart du jéjunum proximal, dans l'espace situé entre les deux ballonnets de la sonde décrite ci-dessus, une injection dans la lumière intestinale de $100 \mu \mathrm{Ci}$ de thymidine méthyle ${ }^{3} \mathrm{H}$ diluée dans $50 \mathrm{ml}$ d'un mélange à parties égales de sérum à 9 p. 1000 ef de glucose isotonique à 5 p. 100, qui est presque entièrement absorbé pendant la durée de l'expérience. Après un temps de contact avec la muqueuse de $1 / 2 \mathrm{~h}$, le liquide restant entre les 2 ballonnets est réaspiré. $A$ l'aide de la sonde à biopsie des prélèvements sont effectués à 48,96 et 120 h. La radioactivité a été mesurée au carbotrimètre SL 40 Intertechnique sur des biopsies prélevées en double exemplaire à 48 et $120 \mathrm{~h}$.

- Le porc 3 a reçu, de la même manière que le porc $2,300 \mu \mathrm{Ci}$ de radio-élément ; le temps de contact avec la muqueuse a duré dans ce cas 1 h 30 . La présence de contenus en quantité importante dans la lumière intestinale chez cet animal a entraîné des difficultés de manipulation et de ce fait seul un prélèvement a pu être effectué à $96 \mathrm{~h}$.

- Pour le porc 4 recevant lui aussi $300 \mu \mathrm{Ci}$ de marqueur, le temps de contact avec la muqueuse a été réduit à $1 \mathrm{~h}$ et des prélèvements ont été opérés à $24,48,72$ et

\section{PLANCHE II}

FIG. 3 et 4. - Histo-autoradiographies. Marquage d̀ la thymidine tritiée. Jéjunum proximal de Porc.

3. $-\times 850 . \mathrm{Fm}:$ position du front de marquage; Bb. bordure en brosse ; $C$, capillaire ; $L$, vaisseau lymphatique.

4. - 1960. Détail de la figure 3 à la base de la villosité ; visualisation des grains d'argent au-dessus des noyaux ayant incorporé la thymidine tritiée. 
PI. II
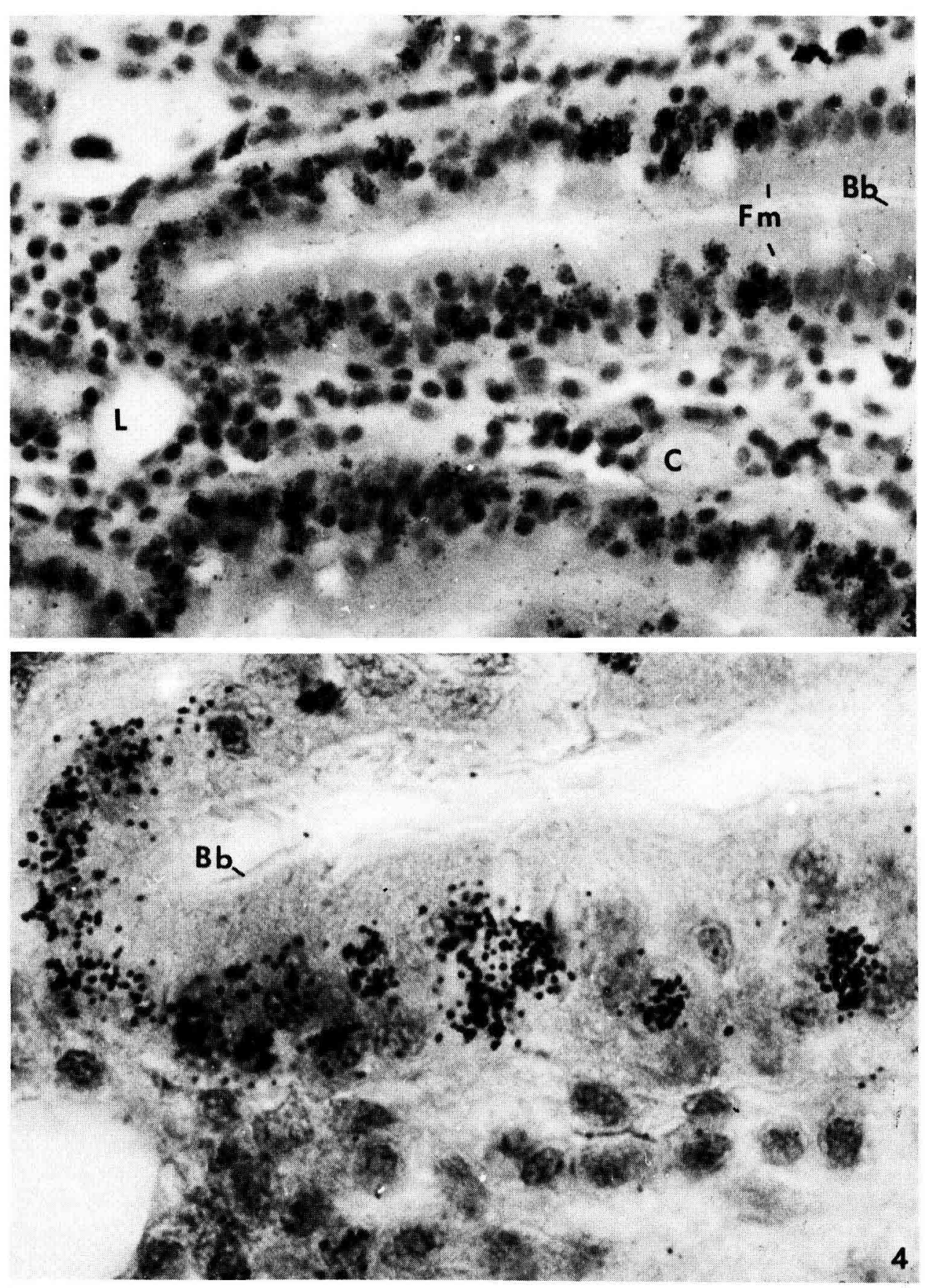
$96 \mathrm{~h}$. La radioactivité contenue dans une biopsie supplémentaire prélevée à $48 \mathrm{~h}$ a été déterminée au carbotrimètre.

\section{Techniques d'histo-autoradiographie.}

Les biopsies sont retirées de la sonde, posées sur un morceau de bristol et orientées les villosités vers le haut (fig. 2). Elles sont immergées dans le mélange fixateur de composition suivante : alcool éthylique absolu 6 vol., formol commercial 3 vol., acide acétique cristallisable 1 vol. Après $6 \mathrm{~h}$ de fixation, les pièces sont déshydratées par passage dans l'alcool butylique pur (4 bains successifs en $24 \mathrm{~h}$ ). L'inclusion est effectuée selon les méthodes classiques. Des coupes sériées sont réalisées sur la totalité des biopsies, la plupart de 5 microns d'épaisseur, quelques autres de $7 \mu$. Elles sont recueillies sur lames gélatinées, puis déparaffinées et recouvertes d'émulsion $K_{2}$ (llford). Après un délai d'exposition de 4 à 4 mois el demi, les préparations sont développées au réfinal (Agfa), rincées, fixées dans l'hyposulfite de sodium et colorées au rouge nucléaire solide picro-indigocarmin (fig. 3 et 4 ).

\section{Mensurations.}

Du fait de l'utilisation de la sonde à biopsie, le prélèvement ne comporte généralement, en plus de la zone des villosités, qu'une partie de la zone des cryptes. Pour cette raison, la position du front des cellules marquées n'est mesurée qu'à partir du collet des cryptes et est exprimée en pourcentage de la hauteur des villosités. Ne sont prises en considération que les villosilés qui sont coupées suivant leur axe, du collet de la crypte au sommet de la villosité, et qui ne présentent alors qu'une seule assise de cellules épithéliales. La visualisation des coupes sériées permet d'éviter de mesurer deux fois la même villosité. Dans la plupart des cas, les biopsies ont été prélevées en double exemplaire, afin d'augmenter l'échantillonnage. En moyenne 10 villosités ont été étudiées pour chaque biopsie.

\section{Résultats.}

Nous exposerons d'abord les résultats concernant les porcs 1 ef 4 , qui nous ont fourni les renseignements les plus détaillés, ensuite ceux du porc 3 et enfin ceux du porc 2.

La figure 5 représente d'une part les moyennes et valeurs extrêmes du front de marquage ( $\mathrm{fm}$ ) aux différents temps des porcs 1 et 4 ef les valeurs expérimentales et la moyenne du porc 3 à $96 \mathrm{~h}$, d'autre part les droites de régression corrèspondant aux porcs 1 et 4 , exprimées respectivement sous la forme temps $=0,88 \mathrm{fm}+17,96$ et temps $=1,21 \mathrm{fm}+12,59$ (les droiles de régression de y par rapport à $x$ sont : porc 1 : $\mathrm{fm}=0.89 \mathrm{t}-8,67$; porc $4: \mathrm{fm}=0,65 \mathrm{t}+0,19)$. Pour les porcs 1 et 4 , le coefficient de corrélation observé est $r=0,88$.

On remarque qu'il existe un écart important entre les valeurs extrêmes du front de marquage quel que soit l'animal étudié et ceci aux différents temps de prélèvement. Aux temps 24,48 et $72 \mathrm{~h}$ communs aux porcs 1 et 4 , cet écart paraît avoir la même importance chez les deux animaux. 
La détermination à partir des données expérimentales des temps qui correspondent à la sortie des cellules marquées du collet des cryptes et à l'arrivée de ces cellules au sommet des villosités fait intervenir les équations des droites de régression : Porc 1 , temps $=0,89 \mathrm{fm}+17,96$; porc 4 , temps $=1,21 \mathrm{fm}+12,59$. Les résultats, affectés de l'intervalle de confiance au risque de 5 p. 100 sont :

- Porc 1 : sortie des cryptes $=18 \mathrm{~h} \pm 2 \mathrm{~h} 50 \mathrm{mn}$; arrivée au sommel des villosités : $106 \mathrm{~h} \pm 11 \mathrm{~h}$ soit 4,4 jours.

- Porc 4 : sortie des cryptes $=12 \mathrm{~h} 36 \mathrm{mn} \pm 6 \mathrm{~h}$; arrivée au sommet des villosités : $133 \mathrm{~h} 36 \mathrm{mn} \pm 18 \mathrm{~h} 36 \mathrm{mn}$ soit 5,5 jours.

- Le Porc 3, traité comme le porc 4, présente des valeurs de front de marquage comprises entre celles des porcs 1 et 4 , plus proches cependant de celles du porc 1 .

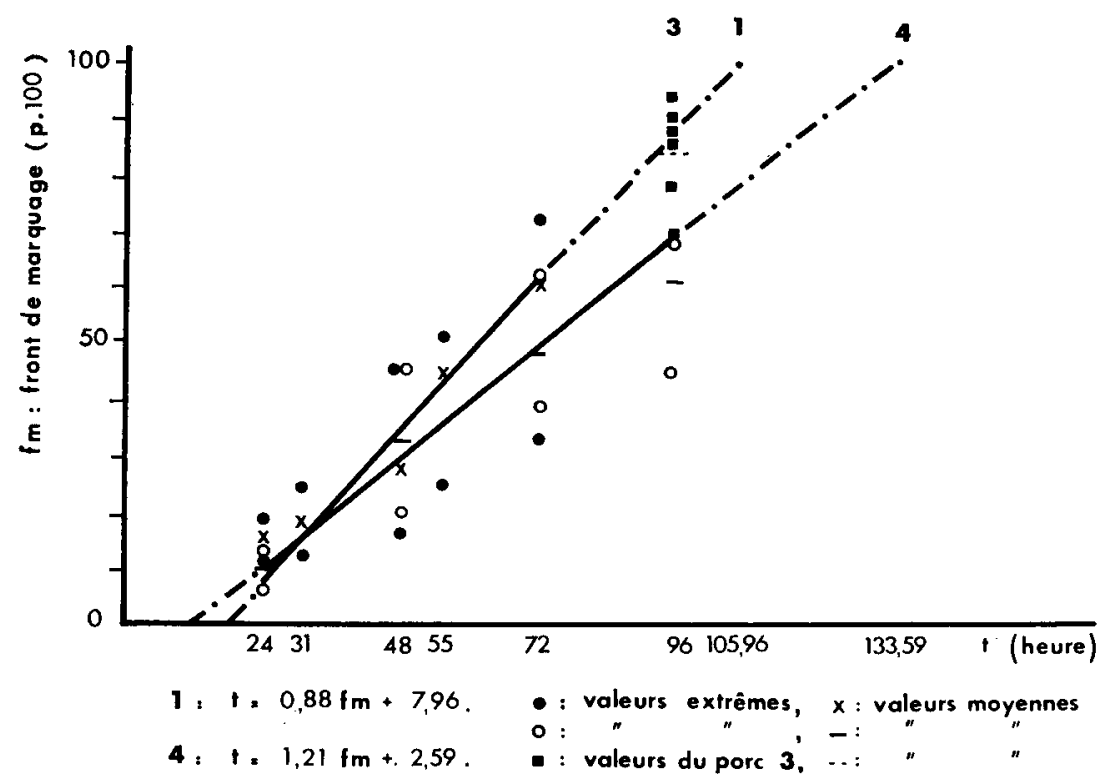

FIG. 5. - Position du front de marquage en fonction du temps, droites de régression temps $=\mathrm{b} \times$ front de marquage + a pour les porcs 1 ef 4 ef valeurs du porc 3

Malgré un délai d'exposition des préparations allant jusqu'à 8 mois, nous n'avons jamais observé de cellules épithéliales marquées chez le porc 2 . Par contre, dès le $4^{\mathrm{e}}$ mois d'exposition, on a observé des lymphocytes très fortement marqués, soit au contact de l'épithélium, soit au niveau du stroma conjonctif des villosités. Les comptages effectués au carbotrimètre, bien que ne précisant pas si la radioactivité se trouve dans l'ADN ef plus particulièrement dans l'ADN des cellules épithéliales, indiquent bien une plus faible radioactivité dans le cas du porc $2(75 \mathrm{dpm} / \mathrm{mg}$ ou $0,06 \mathrm{nCi} / \mathrm{mg}$ de tissu) que dans le cas du porc 3 (770 dpm/mg ou $0,6 \mathrm{nCi} / \mathrm{mg}$ de fissu). Le rendement de comptage pour le tritium est d'environ 60 p. 100. 


\section{Discussion.}

Du fait du mode d'administration du radio-élément, la durée de la perfusion ou celle du temps de contact avec la muqueuse intestinale ne permettent pas d'assigner un temps précis au début du marquage. Ce dernier ne correspond donc pas à un marquage instantané. De plus, l'absence de l'intégrité des cryptes ne permettait pas d'effectuer une mesure précise du point de marquage au temps 0 .

L'utilisation d'animaux porteurs d'une fistule permanente de l'intestin jointe à celle de la sonde à biopsie présente l'avantage d'effectuer l'étude cinétique sur le même animal. On peut cependant se demander dans quelle mesure des biopsies successives influencent le phénomène normal. Le coefficient de corrélation observé de 0,88 indique une bonne corrélation entre le front de marquage exprimé en pourcentage de la hauteur des villosités ef le temps. Comme la majorité des auteurs, nous avons adopté une représentation linéaire du phénomène ; celle-ci ne tient pas compte d'éventuelles variations nycthémérales. En outre, nous n'avons pas cerné l'influence de l'alimentation sur la position des cellules marquées. En effet, les prélèvements effectués le matin portaient sur des animaux à jeun depuis $18 \mathrm{~h}$ et ceux effectués le soir, à $7 \mathrm{~h}$ après le repas du matin. De plus, sur ces animaux qui mangent tous les jours, la présence de contenus en quantité importante au niveau du jéjunum proximal ne nous a pas permis de standardiser les temps de prélèvements. Cela a même conduit à l'obtention d'une seule biopsie pour le porc 3.

Deux explications, au moins, peuvent rendre compte des grandes variations de la position du front de marquage à un temps donné. La première est inhérente au type de prélèvement lui-même, la biopsie ; il est rare de voir plus de 4 à 5 villosités et collets de cryptes adjacentes sur la même coupe. Du fait de l'incidence de coupe, l'on est jamais sûr de passer rigoureusement par le sommet des villosités, ce qui conduit à une valeur de front de marquage en pourcentage de la hauteur des villosités supérieure à la valeur réelle. Cette surestimation fournit une durée de renouvellement plus rapide que ce qui se passe dans la réalité. La deuxième explication est en relation avec la hauteur des villosités observée d'une part sur les biopsies à la loupe avant l'inclusion, d'autre part sur les préparations histologiques. Leur hauteur varie plus pour un site anatomique donné que chez le rat. De plus, les villosités les plus petites n'ont pas systématiquement le front de marquage le plus faible.

La perfusion dans la jugulaire de $0,1 \mu \mathrm{Ci} / \mathrm{g}$ représente une dose suffisante pour assurer un bon marquage. La comparaison des résultats des porcs 2 et 4 permef de dire qu'il faut au moins $300 \mu \mathrm{Ci}$ de thymidine tritiée avec un temps de contact de $1 \mathrm{~h} 30$ lorsque l'injection a lieu dans la lumière intestinale. Dans ce cas, la radioactivité du tissu est de $770 \mathrm{dpm} / \mathrm{mg}$ soit environ $0,6 \mathrm{nCi} / \mathrm{mg}$ de tissu. Le temps de contact peut d'ailleurs être ramené à $1 \mathrm{~h}$ (Porc 3).

L'intérêt incontestable de ce procédé de marquage local réside dans l'utilisation d'une très faible quantité de radio-élément, $300 \mu \mathrm{Ci}$ pour un porc de $55 \mathrm{~kg}$, soit la quantité utilisée pour un rat de $300 \mathrm{~g}(1 \mu \mathrm{Ci} / \mathrm{g})$; la présence d'une fistule permanente de l'intestin et l'utilisation de la sonde à biopsie, permettent d'effectuer une étude cinétique sur le même animal, fait important chez le porc où il est difficile d'effectuer des expériences en lots. Par contre, cette faible quantité entraîne une durée d'exposition 
des préparations de l'ordre de 4 mois et demi. Les quelques coupes effectuées à $7 \mu$, du fait d'une plus grande quantité de radio-élément dans la préparation histologique, permettent une durée d'exposition plus courte (environ 3 mois). L'épaisseur de 5 microns, utilisée pour la majorité des coupes de ce travail représente donc un bon compromis entre une durée d'exposition des préparations non démesurément allongée et une définition satisfaisante de la position du front des cellules marquées. Elle exclut cependant une étude plus précise, par exemple l'évaluation d'un index de marquage, ce qui n'était d'ailleurs pas le but du présent travail.

Compte tenu des restrictions précédemment citées et du petit nombre d'animaux étudiés, la durée du renouvellement de l'épithélium intestinal de 4 jours et demi à 5 jours et demi déterminée dans nos conditions d'expérience ne peut être étendue à un ensemble de porcs d'un an. Des informations complémentaires seront donc nécessaires. Moon indique que l'épithélium des villosités de l'intestin grêle est renouvelé en 7 à 10 jours chez les porcelets âgés d'un jour, tandis qu'il est remplacé en 2 à 4 jours chez les porcelets de 3 semaines. Si l'on tient compte de l'influence du vieillissement qui se traduit par un ralentissement du renouvellement de l'épithélium intestinal (Buetow, 1971), les données que nous indiquons représentent un bon ordre de grandeur de la durée du phénomène.

Accepté en juillet 1976.

Remerciements. - Nous exprimons nos remerciements à P. A. Villiers du Laboratoire de Radiobiologie Appliquée pour la réalisation des fistules intestinales permanentes et l'utilisation de la sonde à biopsie.

\section{Références}

BUETOW D. E., 1971. Chapter 4. Cellular content and cellular proliferation changes in the tissue and organs of the aging mammal. $V$. Changes in the mucosal cells of the intestine : 97-106 in CAMERON, I. L. and THRASHER, J. D. Cellular and molecular renewal in the mammalian body, Academic Press.

MAWHINNEY B. S., AUSTIN, B. T., RILEY E. F. Jr., 1972. The uptake of topically applied tritiated thymidine by chick embryos. Proc. Soc. Exp. Biol. Med., $140: 208-211$.

MOON H.W., 1971. Epithelial cell migration in the alimentary mucosa of the suckling pig. Proc. Soc. Exp. Biol. Med., $137: 151-154$.

MOON H. W, JOEL D. D., 1975. Epithelial cell migration in the small intestine of sheep and calves. Am. J. vet. Res., $36: 187$.

WACHHOLZ B.W., HOPPER A., 1971. Labeling of the intestinal mucosal cell by intraluminally administered ${ }^{3} \mathrm{H}$ thymidine. Rapport américain. BNWL 1550, Part 1 : 15-16. 